Trascender, Contabilidad y Gestión. Vol. 7, Núm. 19 (enero - abril del 2022).

Universidad de Sonora. Departamento de Contabilidad. México.

ISSN: 2448-6388. Reserva de Derechos 04-2015-04172070800-203.

\title{
El fideicomiso público estatal del impuesto de fomento al turismo: Identificación en la estructura de la red del flujo financiero y su exigua transparencia
}

\author{
The state public trust of the tourism promotion tax: Identification in the financial flow \\ network structure and its meager transparency
}

Esthela Carrillo Cisneros ${ }^{1}$; Alejandro Arnulfo Ruiz León ${ }^{2}$; Luis Enrique Ibarra Morales ${ }^{3}$

Recibido: 28 de agosto de 2021.

Aceptado: 4 de diciembre de 2021.

DOI: https://doi.org/10.36791/tcg.v7i19.116

JEL: H7. Administración estatal, autonómica y local; Relaciones intergubernamentales.

H72. Presupuesto y gasto de la administración estatal, autonómica y local.

H83. Administración Pública, Sector Público.

\section{Resumen}

El impuesto de hospedaje, gravamen indirecto que incide en el desarrollo económico local al retornar dicho impuesto a través de promoción y publicidad turística. Relevantes comportamientos de mecanismos administrativos en el operar las finanzas públicas con necesidad de mayor transparencia y de acceso a

\footnotetext{
${ }^{1}$ Esthela Carrillo Cisneros. Contador Público. Maestra en Impuestos. Doctora en Ciencias Administrativas (UACJ). Profesor investigador de tiempo completo en Academia de Contabilidad, Finanzas y Administración. Universidad Estatal de Sonora (UES), México. Correo: esthela.carrillo@ues.mx. Correo alterno: esthela7carrillo@gmail.com. ORCID: https://orcid.org/0000-0002-5401-9394.

${ }^{2}$ Alejandro Arnulfo Ruiz León. Licenciatura en Actuaría. Maestro en Bibliotecología y estudios de la información. Profesor en el Instituto de Investigaciones en Matemáticas Aplicadas y en Sistemas en Universidad Nacional Autónoma de México (UNAM), Coyoacán, Distrito Federal, México. Correo: rarnulfo@unam.mx. Correo alterno: rarnulfo@gmail.com. ORCID: https://orcid.org/0000-0002-4543-6874.

${ }^{3}$ Luis Enrique Ibarra Morales. Ingeniero Industrial y de Sistemas. Maestro en Administración. Doctor en Filosofía con Especialidad en Administración (UANL-FACPyA). Profesor de tiempo completo en la Universidad Estatal de Sonora, Sonora, México. Correo: luis.ibarra@ues.mx. Correo alterno: luisim00@hotmail.com. ORCID: https://orcid.org/0000-0001-8804-3934.
}

Carrillo Cisneros, E., Ruiz León, A. A., e Ibarra Morales, L. E.
Vol. 7, núm. 19 / enero - abril del 2022 DOI: https://doi.org/10.36791/tcg.v7i19.116

Pp. 88-106 
información, propicia generar este trabajo, siendo el fideicomiso público estatal un nodo con necesidad de someterse a estricta rendición de cuentas del destino y uso de este impuesto.

Para esto, el objetivo es mapear las relaciones de colaboración y actores obligados partícipes en el flujo de recursos financieros para identificar la figura del fideicomiso público como elemento relevante de transparencia de política pública. En la metodología se aplica el Análisis de Redes de Sociales con categoría teórica redes de políticas públicas; se apoya con el método inductivo y de tipo longitudinal.

Hay avances y consolidaciones para informar respecto a los recursos públicos estatales comprometidos en fideicomisos, resultando fundamental reconocer la aplicación de los marcos normativos vigentes correlacionados. La red permitió observar objetivamente el espectro de los sujetos públicos y privados obligados a transparentar el ejercicio de los recursos y gradual alineación en la implementación de reportes financieros de la política pública estatal contextual a este estudio.

Palabras clave: fideicomiso, transparencia, impuesto hospedaje.

\begin{abstract}
The lodging tax, an indirect tax that affects local economic development by returning the mentioned tax through tourism promotion and advertising. Relevant behaviors of administrative mechanisms in the operation of public finances with the need for greater transparency and access to information, favors generating this job, being the state public trust a node in need of submitting to strict accountability of the destination and use of these resources.
\end{abstract}

For this, the objective is to map the collaborative relationships and obligated actors involved in the flow of financial resources to identify the public trust figure as a relevant element of public policy transparency. In the methodology, the Social Networks Analysis is applied with the theoretical category of public policy networks; It is supported by the inductive method and longitudinal type.

There are advances and consolidations to inform regarding the state public resources committed in trusts, it is essential to recognize the application of the correlated normative frameworks in force. The network allowed to objectively observe the spectrum of public and private subjects obliged to make the exercise of resources transparent and gradual alignment in the implementation of financial reports of the state public policy contextual to this study.

Keywords: trust, transparency, lodging tax.

\section{Introducción}

De relevancia la reforma al artículo seis de la Ley General de Transparencia y Acceso a la Información Pública (LGTAIP) en vigor, donde se destaca para este trabajo que dichos cambios contemplaron la incorporación de sujetos obligados que antes no se habían incluido en materia de transparencia y rendición de cuentas, como aquellos involucrados en la utilización de bienes del Estado o hechos relacionados en cualquier acto público. Es a través de esa ley, que se obliga a los fideicomisos públicos de cualquier ámbito (federal, estatal o municipal), al cumplimiento en materia de transparencia y acceso a la información.

Aquí en particular, se examina la figura jurídica del fideicomiso público estatal que interviene como actor administrador durante el flujo del recurso financiero del Impuesto por la Prestación de Servicio de Hospedaje 
(IPSH), el cual se constituye con el propósito de auxiliar en las atribuciones del Estado a través de un comité técnico como órgano responsable que debe operar bajo reglas y lineamientos cuyas decisiones deben ser discutidas, valoradas y consensuadas, a fin de dedicar gestión transparente en las erogaciones o aplicación del impuesto, integrando facultades relacionadas con el objeto de creación: canalizar dichos recursos a incentivar el turismo que finalmente incide en desarrollo económico local.

En el diagnóstico de transparencia y monitoreo de los fideicomisos públicos, se explica que "son vehículos financieros que se formalizan a través de contratos, para [..] acciones gubernamentales específicas [..] para la implementación de política pública, por lo que el destino de sus recursos debe ser rastreable al cumplimiento de objetivos socialmente útiles” (Martínez, 2020, p.21).

Evidentemente que le corresponde a la entidad atender el cumplimiento a lo dictado en el marco normativo contextual para evitar opacidad en el cumplimiento del objeto del contrato de fideicomiso a través del cual se administra el gasto público; sin eximir a cada una de las partes de las responsabilidades que les correspondan.

En este trabajo de investigación fue sinuoso el camino para acceder a información financiera, sobre todo del destino de los recursos. No obstante la Organización para la Cooperación y Desarrollo Económicos (OCDE, 2016) señala que "los datos abiertos son parte integral de los esfuerzos en el fortalecimiento de la productividad, la rendición de cuentas y la integridad del gobierno, para facilitar la innovación cívica y empresarial, y promover la formulación de políticas basadas en evidencia”.

Hay avances y consolidaciones en materia de transparencia y rendición de cuentas respecto a los recursos públicos comprometidos en fideicomisos federales pero que en algunos de los fideicomisos estatales está parsimonioso el proceso; pero se trata de un problema mundial, no solo de una entidad federativa, por lo que con este trabajo de investigación se aportan realidades de dicha situación.

\section{Objetivo}

Mapear las relaciones de colaboración y actores obligados partícipes en el flujo de recursos financieros para identificar la figura del fideicomiso público estatal como elemento relevante de transparencia de política pública.

\section{Antecedentes}

Este trabajo es continuación del proyecto de investigación enfocado al análisis integral del Impuesto al Servicio de Hospedaje, tributo local en la entidad federativa donde se identificó e integró la red de colaboración pública-privada-social a través del funcionamiento de las redes de políticas públicas (policy network) que coexisten en el flujo financiero del tributo, considerando la tendencia a que la transparencia se fortalezca en la administración del citado impuesto.

Parte de las conclusiones de Carrillo, et al. (2019) del estudio previo, fue que limitaciones estructurales en la red se hacen presentes como nodos de poder en función de la conveniencia del flujo financiero del impuesto y en relación a la aplicación del mismo; ya que este impuesto se administra a través de la figura del fideicomiso; por lo que "el intercambio de recursos es el principal cimiento de las relaciones entre los actores, es una precondición de las redes de política pública” (Klijn, 997).

En las redes de política, destacan los siguientes conceptos clave de Jung (2010):

Triángulo de acero. Conformado por grupos de interés, comités y agencias ejecutoras.
Vol. 7, núm. 19 / enero - abril del 2022

DOI: https://doi.org/10.36791/tcg.v7i19.116

Pp. 88-106 
Redes de asuntos. Concepto muy cuestionado y al que a veces se pretende sustituir por el de remolinos, refiriéndose a aquellas redes conformadas por un elevado número de participantes que incluyen intereses tanto individuales como grupales.

Comunidades de política. En ellos se combina la amplitud de participantescomo en las redes de asuntos-, pero se reconoce también una larga durabilidad de las relaciones, la que es también característica en los triángulos de acero.

Coaliciones de defensa. Son grupos de coaliciones que compiten entre sí y están conformados por entre dos y cuatro componentes $\mathrm{y}$, cada uno, tiene una variedad de actores públicos y privados que comparten creencias comunes.

Antes de resaltar la importancia de la figura jurídica del fideicomiso como elemento en la administración del recurso financiero del impuesto, es necesario contextualizarlo, donde en la generalidad de las entidades federativas estos ingresos se utilizan para promover los destinos turísticos. En Sonora se establece que el 90\% recaudado debe retornarse a través de promoción y publicidad turística, y el restante $10 \%$ se debe destinar a la administración y fiscalización, de acuerdo con lo establecido en la ley de hacienda del estado vigente.

Dicha recaudación, representa un importante ingreso para las entidades federativas y para las haciendas públicas es uno de los principales impuestos locales. Como se puede observar en el gráfico 1, de acuerdo con los ingresos de impuestos estatales nacionales publicados a través de las finanzas públicas estatales por el Instituto Nacional de Estadística y Geografía (INEGI), del ejercicio fiscal 2019, después del impuesto sobre nóminas y el impuesto sobre tenencia o uso de vehículos, la recaudación del impuesto sobre hospedaje está entre los de mayor presencia nacional.

\section{Gráfico 1}

Impuestos estatales de mayor recaudación a nivel nacional

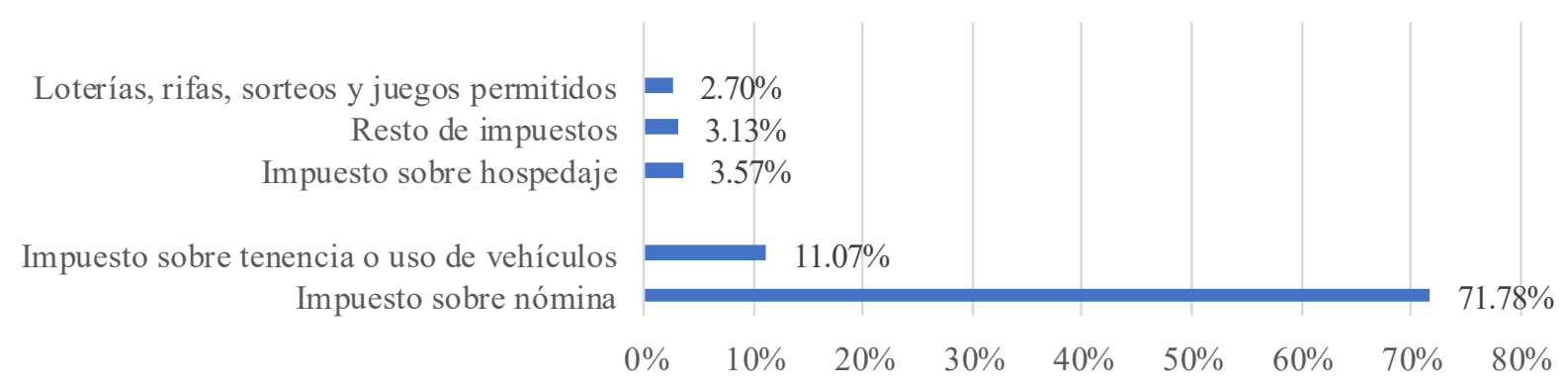

Fuente: Elaboración propia con datos de finanzas públicas estatales emitidas por INEGI (2021). 
Integrando la recaudación nacional del citado impuesto durante cinco años (2015 al 2019), se pueden identificar en la siguiente imagen 1, a las principales entidades federativas con mayores ingresos, siendo Quintana Roo con el 41.09\%, Baja California Sur con 9.55\%, Jalisco 8.13\% y Nayarit 5.14\%. Sonora se ubica en $15^{a}$ posición con el $1.38 \%$.

\section{Imagen 1}

Entidades federativas con mayor recaudación de impuesto sobre hospedaje (nivel nacional 2019-2015)

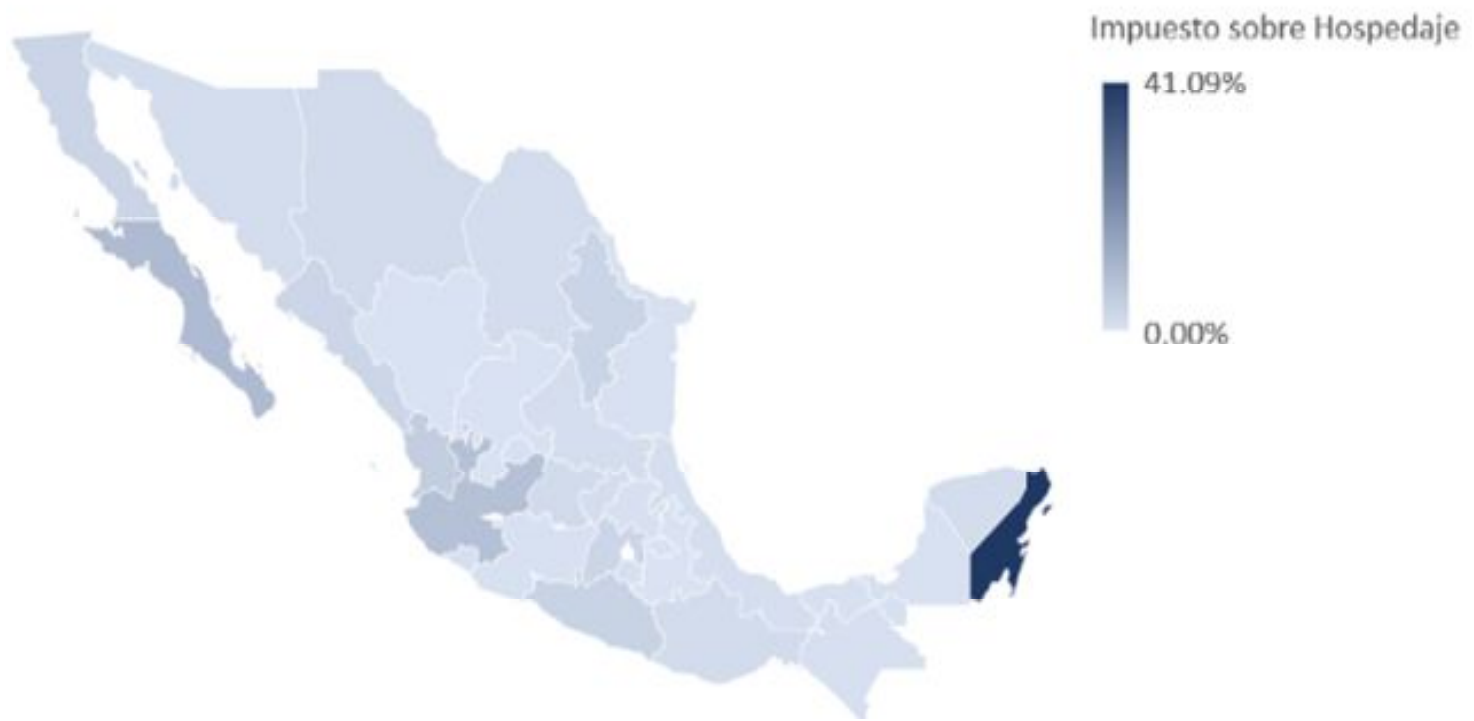

Fuente: Elaboración propia con datos de finanzas públicas estatales de INEGI (2021). Ingresos sobre Impuesto Hospedaje durante 2015-2019.

Es importante especificar que la tasa de recaudación de este impuesto no es homogénea, la cual varía desde un $2 \%$ hasta un $7 \%$, considerando que hay estados con mayor afluencia turística que otros; aunado a que México ha estado creciendo como destino turístico de manera extraordinaria, lo que directamente beneficia en derrama y desarrollo económico coadyuvado al retorno de la contribución. En cifras locales, la recaudación del impuesto al hospedaje depende directamente de las variables cuartos ocupados y turistas noches; además de compararse con el impuesto presupuestado que año tras año se pretende alcanzar; comportamiento que a través de los años se representa de forma ascendente.
Carrillo Cisneros, E., Ruiz León, A. A., e Ibarra Morales, L. E.
Vol. 7, núm. 19 / enero - abril del 2022 DOI: https://doi.org/10.36791/tcg.v7i19.116

Pp. 88-106 


\section{Gráfico 2}

Comportamiento del Impuesto al Hospedaje recaudado y presupuestado del 2015 al 2019, con estadística de turista noche y cuartos ocupados

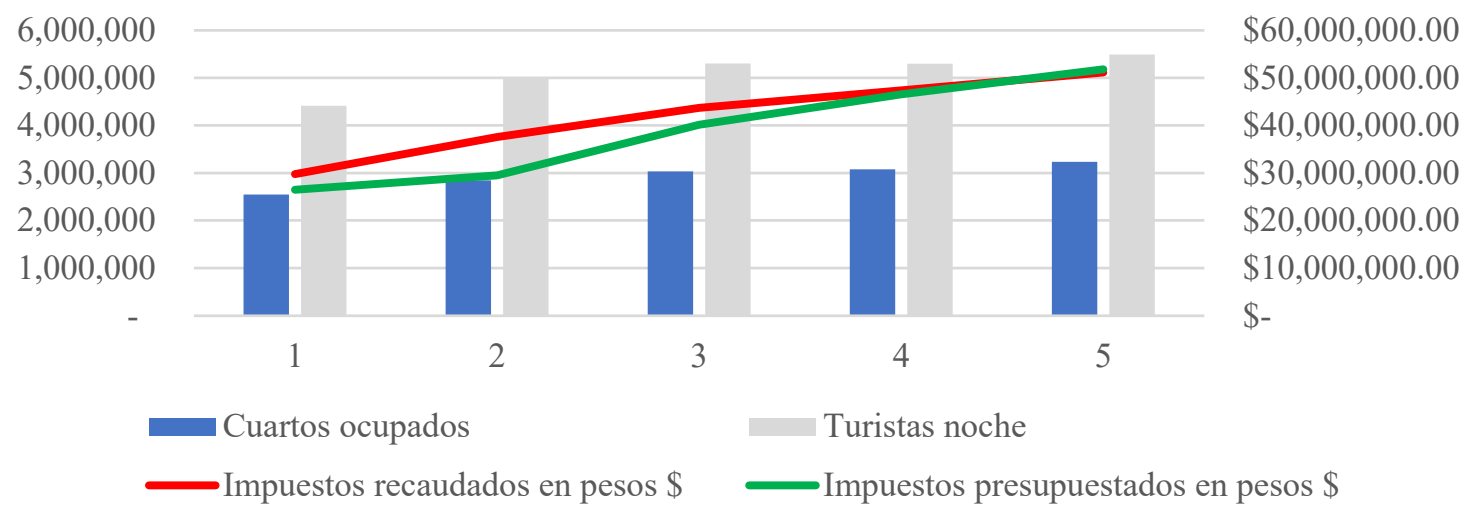

Fuente: Elaboración propia con cifras de las finanzas públicas estatales emitidas por INEGI, 2021.

Las cifras anteriores se representan a través de la correlación de Pearson con datos de cinco años, donde resulta con alta correlación entre dichas variables como abajo se relaciona.

\section{Tabla 1}

\section{Correlaciones Pearson entre variables}

\begin{tabular}{lccc} 
Impuesto & $\begin{array}{c}\text { Impuesto } \\
\text { Presupuestado }\end{array}$ & $\begin{array}{c}\text { Impuesto } \\
\text { Recaudado }\end{array}$ & $\begin{array}{c}\text { Turistas } \\
\text { Noche }\end{array}$ \\
\cline { 2 - 4 } Presupuestado & & & \\
\hline Impuesto Recaudado & $.971^{* *}$ & & \\
\hline Turistas Noche & $.899^{*}$ & $.974^{* *}$ & $.991^{* *}$ \\
\hline Cuartos OCupados & $.942^{*}$ & $.993^{* *}$ & \\
\hline
\end{tabular}

Fuente: Elaboración propia con datos de INEGI (2021) y cifras turísticas emitidas por Secretaría de Turismo (2021). 
En contexto, las acciones fiscales COVID-19, como parte de las medidas y programas para apoyar a la población de la entidad federativa de Sonora, se concedió la exención temporal del $100 \%$ en el pago de impuestos por la prestación de servicios de hospedaje durante el ejercicio fiscal 2020, lo que se verá reflejado en caída de ingresos en las próximas finanzas públicas.

Y bien, las cifras anteriores justifican la relevancia de la presencia del impuesto, por lo mismo, deben tender a ofrecer transparencia en la administración del mismo, contexto que se resalta en este trabajo, ya que fue lento el camino para acceder a las finanzas públicas estatales con solicitudes de información a través del Sistema de Solicitudes de Información Pública del Estado de Sonora (INFOMEX) y de consulta pública a través de la Plataforma Nacional de Transparencia (PNT) para conocer las erogaciones del impuesto. La información disponible en portales estatales, hasta la fecha de este documento, ha resultado insuficiente y escasamente desagregada para su interpretación, además de tiempo extendido al declinarse por la autoridad hacendaria algunas solicitudes o delegarse a la COFETUR y OCV's las comprobaciones financieras.

Tomando en cuenta que la exposición de motivos al decretar leyes fiscales justifica la existencia y permanencia de las contribuciones, también es necesario justificar y explicar en un contexto de transparencia y acceso a la información las erogaciones y propósitos a los que se destinan las recaudaciones, en cuyo proceso administrativo resalta la figura del fideicomiso público estatal.

\section{Fideicomiso Público}

El fideicomiso antecede de 1932 como una operación de derecho privado en la Ley General de Títulos y Operaciones de Crédito (LGTOC), actualmente vigente (Suayeta, 2002).
Desde entonces, la LGTOC vigente y a partir del artículo 381 regula esta figura, considerándola como contrato jurídico mercantil y financiero, citándose que:

En virtud del fideicomiso, el fideicomitente transmite a una institución fiduciaria la propiedad o la titularidad de uno o más bienes o derechos, según sea el caso, para ser destinados a fines lícitos y determinados, encomendando la realización de dichos fines a la propia institución fiduciaria (LGTOC, 2021).

Para el sector público, derivan otros lineamientos vigentes al contexto emitidos a través del Diario Oficial de la Federación, en cuyo decreto vigente se establecen los lineamientos que deberán observar las dependencias y entidades de la administración pública federal para el control, la rendición de cuentas e informes y la comprobación del manejo transparente de los recursos públicos federales otorgados a fideicomisos, mandatos o contratos análogos.

En complemento se ha emitido el Acuerdo vigente que establece los Lineamientos que deberán observar los entes públicos para registrar en las cuentas de activo los fideicomisos sin estructura orgánica y contratos análogos, incluyendo mandatos.

\section{Revisión del Marco normativo de los fideicomisos públicos}

Debido a que no existe la exclusividad a una sola ley que regule los fideicomisos públicos, se concentra en la siguiente imagen 2 gran parte del marco normativo, el cual sirve de referencia para consultar aspectos de su administración, operación y constitución, que como se observa, hay interacción tripartita en la aplicación de esta figura: Presupuestal, Regulación Básica y en materia de Transparencia y Acceso a la información.
Vol. 7, núm. 19 / enero - abril del 2022

DOI: https://doi.org/10.36791/tcg.v7i19.116

Pp. 88-106 


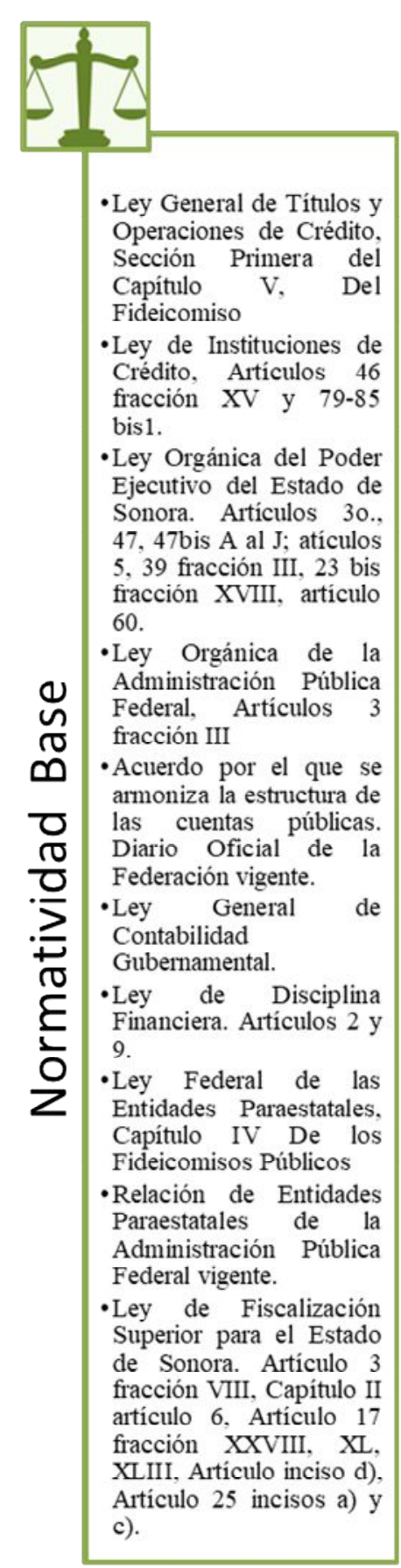

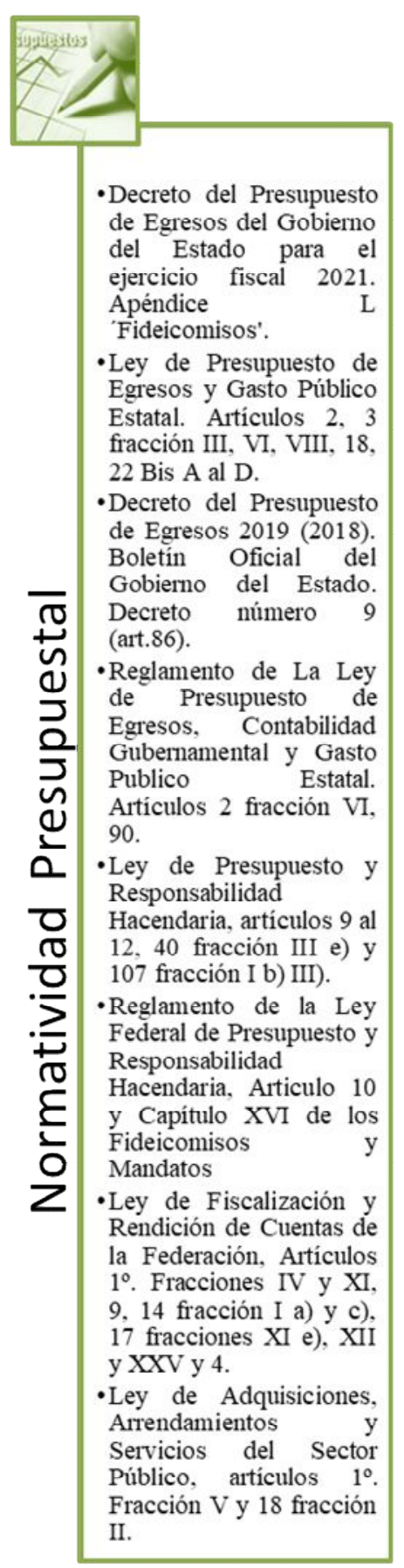

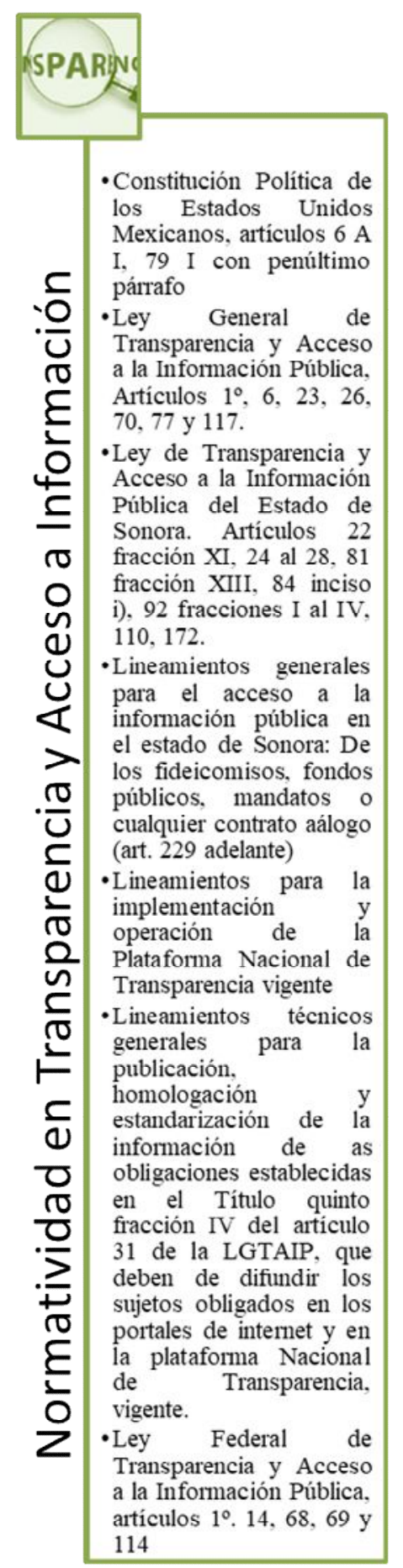

Fuente: Elaboración propia con análisis al marco normativo que regula al fideicomiso. 


\section{Transparencia y Rendición de cuentas en fideicomisos públicos}

En lo que se refiere a la administración de los recursos públicos, ésta se encuentra plagada de esquemas técnicos y jurídicos que hacen de su seguimiento y control un ejercicio complicado para el grueso de la población e incluso para la sociedad civil organizada y los estudiosos de la materia. (Salvatierra, 2019, p. 9).

La falta de transparencia en la administración de diversos fideicomisos públicos ha sido resultado de omisiones, imprecisiones o incluso interpretaciones a conveniencia del marco normativo aplicable a dicho instrumento jurídico, conllevando con ello actos de corrupción, por lo que se han observado recientes reformas constitucionales y específicamente al artículo seis, referente a la transparencia y acceso a la información.

Para expresar con cifras lo anterior, se antecede estudio realizado por la organización mexicoevalua.org (2021), el cual expone antecedentes observados que están destapando el actual proceso de extinción de diversos fideicomisos, donde al 1er trimestre del ejercicio fiscal 2020, la autoridad hacendaria reportó 332 actos jurídicos registrados, de los cuales 303 son fideicomisos, 23 mandatos y 6 análogos, que en total ascienden a \$728.4 mmdp. “los recursos disponibles se utilizarían para contribuir al equilibrio presupuestario de aquellos programas que determinara la SHCP; sin embargo, no se tiene registro del destino y uso de esos recursos”.

Por otra parte, estudio realizado por la Asociación Nacional de Cadenas de Hoteles (ANCH) y la Asociación Mexicana de Hoteles y Moteles (AMHM), identifican en su trabajo ingresos recaudados $\mathrm{y}$ transferidos a fideicomisos y aportaciones a las Asociaciones de Hoteles y Moteles. Los hallazgos más relevantes fueron:
[..] En la mayoría de los casos, lograr el acceso a la información requerida supuso la solicitud a varias autoridades que se declararon incompetentes y la interposición de recursos de revisión ante las autoridades en materia de transparencia correspondientes, lo cual implicó largos periodos de tiempo y esfuerzo.

[...] Ninguna entidad entregó la información con el grado de detalle que se solicitó. Después de presentados y ganados los recursos de revisión, entregaron la información con el detalle requerido.

De lo anterior, propusieron a todas las Oficinas de Convenciones y Visitantes del país que comiencen a utilizar la Ley General de Transparencia y Acceso a la Información Pública, para solicitar cada año la información que requieren para operar los planes de mercadeo y monitorear con esa herramienta si están recibiendo los recursos que por ley debieran destinarse a la promoción de sus destinos (Asociación Nacional de Cadenas de Hoteles, 2014).

Todo el contexto anterior, acontece en la decisión del Ejecutivo al emitir el Decreto por el que se ordena la extinción o terminación de los fideicomisos públicos, mandatos públicos y análogos, emitido el 2 de abril de 2020 en el Diario Oficial de la Federación, lo que impacta en una forma de entender lo público "los fideicomisos públicos están sujetos a la transparencia, rendición de cuentas, fiscalización y responsabilidades; si no se agotan esas variables, quedará la sensación que se determinó extinguir una herramienta útil de la administración pública mexicana por razones estrictamente extrajurídicas”. En complemento, el investigador añade que "si se prueban irregularidades en la extinción de los fideicomisos, existen omisiones punibles; en ese sentido también se deben imputar
Carrillo Cisneros, E., Ruiz León, A. A., e Ibarra Morales, L. E.
Vol. 7, núm. 19 / enero - abril del 2022 DOI: https://doi.org/10.36791/tcg.v7i19.116

Pp. 88-106 
responsabilidades a quienes tenían la obligación de vigilar y controlar a los fideicomisos públicos" (Márquez, 2020).

\section{Método}

El método utilizado es Análisis de Redes de Sociales ARS (Social Network Analysis), con la categoría teórica de redes de gobernanza o redes de políticas públicas (Public Policy Networks). En este caso, fue necesario integrar la red de colaboración e identificación de los actores que intervienen particularmente en el proceso de flujo de recursos financieros del impuesto y su relación con el fideicomiso público.

Las aproximaciones que se obtienen en el mapeo de relaciones, resultan de vínculos y nodos que están basados en datos obtenidos de entrevistas, análisis exhaustivo al marco normativo aplicable, análisis cuantitativo a datos de finanzas públicas y consultas de información pública a través del Sistema de Solicitudes de Información Pública del Estado (INFOMEX) y la Plataforma Nacional de Transparencia (PNT), además de estudio previo realizado por Carrillo, et al. (2019) donde se modelo estructura reticular general del proceso. Esta investigación se complementa con el tipo de investigación explicativa y no experimental, pues "establecen relaciones de causalidad entre hechos, conceptos y variables en un contexto concreto, generando un sentido de entendimiento de problemas que se examinan” considerando además que el estudio es de carácter longitudinal "sirven para efectuar observaciones en dos o más momentos o puntos en el tiempo", que, en este caso, se observaron los procesos y flujos durante los ejercicios fiscales 2015 al 2020 (Hernández, et al. 2018, p.188). Se complementa el estudio con el método inductivo.

\section{Supuesto}

La figura jurídica del fideicomiso público estatal del $2 \%$ de hospedaje se percibe como un nodo con necesidad de transparencia en la estructura de la red, ya que depende directamente de la responsabilidad de las partes intervinientes, de la calidad que la política pública es ejecutada y de si se logran resultados en la población objetivo.

\section{Resultados y Discusión}

Se logró el mapeo de relaciones e identificación de colaboraciones que conlleva el flujo de recursos financieros del impuesto al servicio de hospedaje; por lo que se da cumplido el objetivo planteado en esta investigación. Es durante dicho proceso que se identificó al fideicomiso público como actor directamente relacionado y obligado a intervenir a lograr un uso eficaz, eficiente y transparente de los recursos financieros del impuesto, previo lo autorizado por la autoridad hacendaria, comité técnico y comité de administración a cargo de las entidades turísticas a cargo de ejercer el recurso.

En la siguiente estructura reticular se identifica de izquierda a derecha el flujo que sigue la captación del impuesto. Los componentes básicos de la red son las instituciones (nodos) que en ella participan, las cuales adquieren una serie de atributos reticulares en un medio de intercambio y dependencia de recursos financieros. La construcción de la red está basada en una matriz de relaciones previamente creada y obtenida de diversas fuentes. Con el estudio a detalle del flujo que sigue la recaudación del impuesto, se construyó la matriz de relaciones. 


\section{Imagen 3}

Estructura reticular del flujo financiero del impuesto al hospedaje y su fideicomiso público

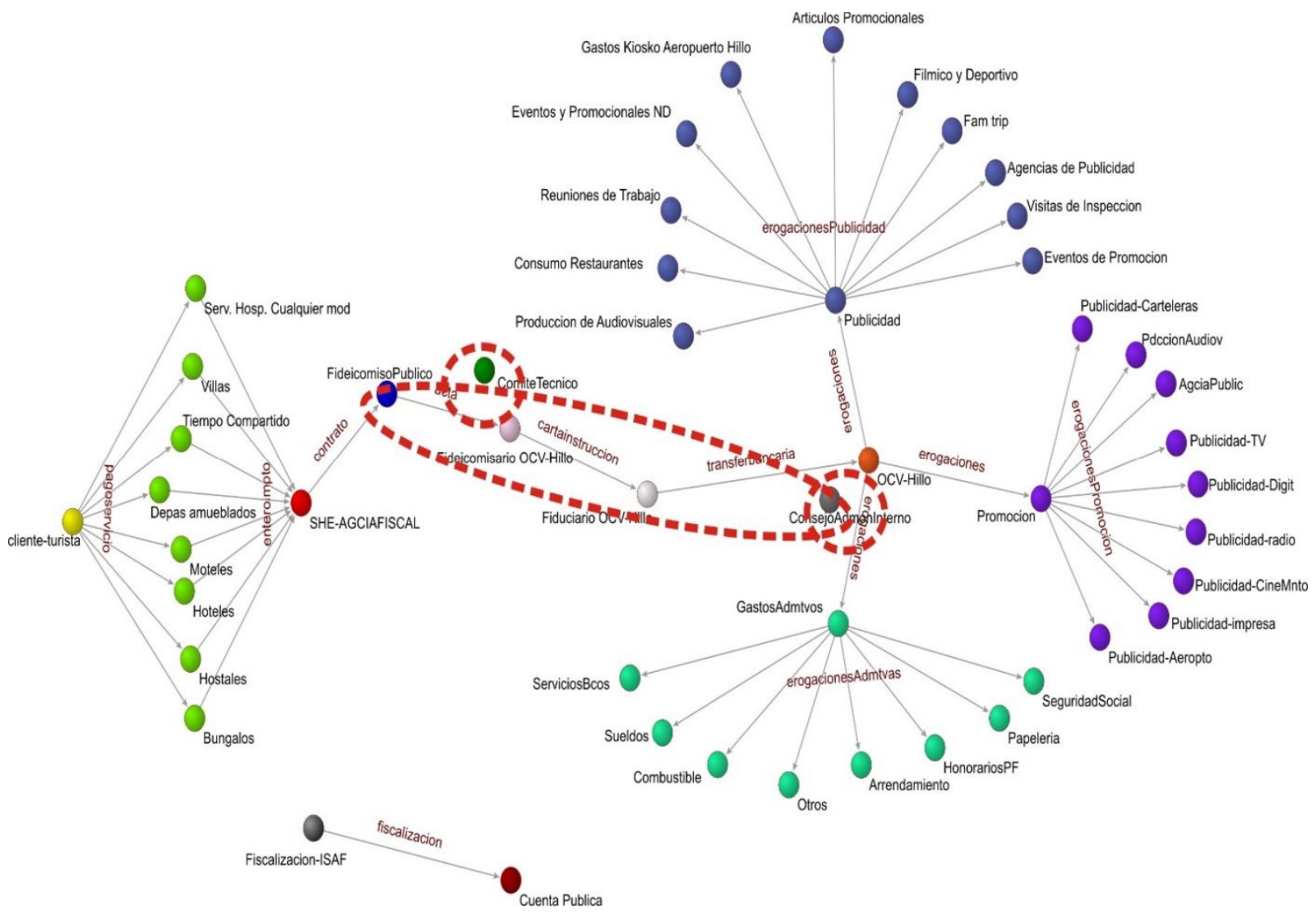

Fuente: Elaboración propia de Carrillo, E. y Ruiz, A., (2021) con datos de investigación procesados en software Pajek V.5.12.

\section{Discusión}

La discusión es explicativa-inductiva a partir de los resultados de la investigación. La estructura reticular (imagen 3) inicia de izquierda a derecha con el nodo amarillo [cliente-Turista] a quien se le aplica la tasa del impuesto del $2 \%$ sobre el valor de la contraprestación que recibe por el servicio de hospedaje. Posterior el contribuyente [Hoteles, moteles y otras modalidades], efectúan el traslado del impuesto a la autoridad hacendaria (nodo rojo).
En la red se identifican los nodos (bajo línea punteada con rojo) que corresponden a la participación del fideicomiso público del impuesto al $2 \%$ de hospedaje en la entidad federativa. Como se observa, su acción es previa a las erogaciones aplicadas en promoción y publicidad turística y también previa la autorización del comité técnico y consejo de administración.

El contrato de fideicomiso público de administración e inversión del impuesto está celebrado entre el Gobierno del Estado, a través del Secretario de Hacienda
Carrillo Cisneros, E., Ruiz León, A. A., e Ibarra Morales, L. E.
Vol. 7, núm. 19 / enero - abril del 2022

DOI: https://doi.org/10.36791/tcg.v7i19.116

Pp. 88-106 
[fideicomitente y Fideicomisario] y la institución de Banca Múltiple [Fiduciario].

Al interior del contrato se formalizan a los servidores públicos e integrantes del sector turístico privado integrantes del Comité Técnico, el cual está legalmente indicada su integración en la Ley de Hacienda del Estado:

\section{Imagen 4}

Integrantes del Comité Técnico

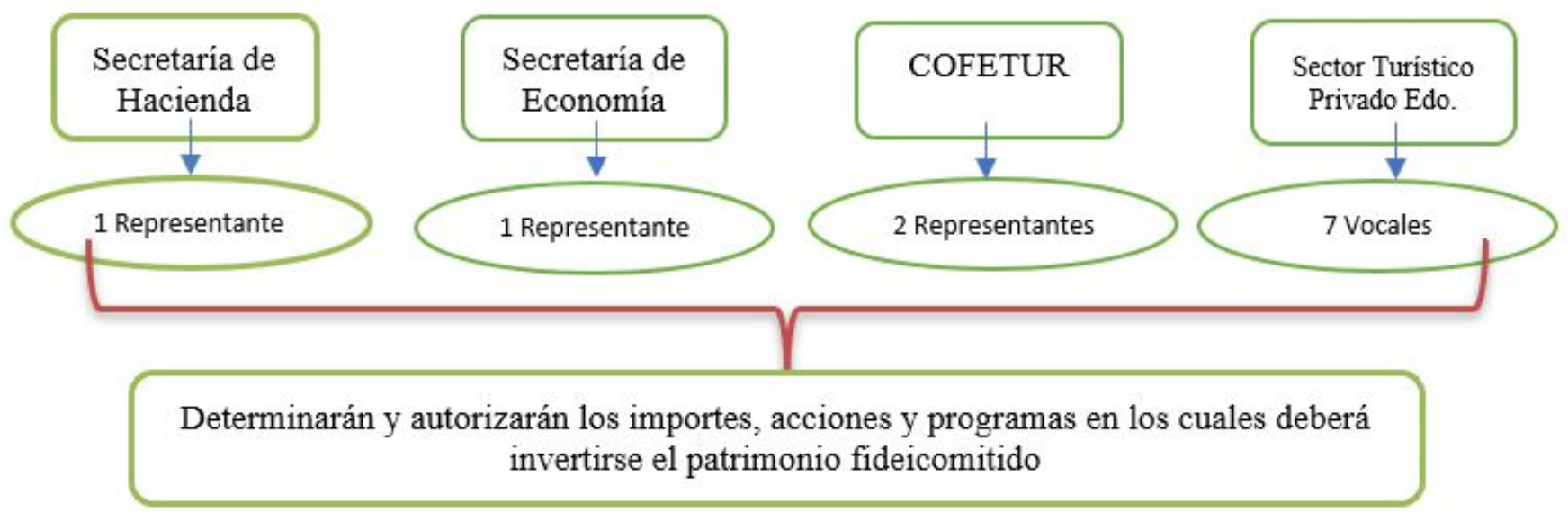

Fuente: Elaboración Propia con datos de Contrato de Fideicomiso público y Ley de Hacienda del Estado (2021).

Una vez que llega el recurso financiero a las Oficinas de Convenciones y Visitantes (OCV), interviene otro nodo llamado Consejo de Administración, quienes también determinan y autorizan importes acciones y programas dentro de las partidas de promoción y publicidad turística. La identificación de los distintos tipos de gastos que llevan a cabo las OCV como se observa su desglose en la red, fue posible al exhibir información contable dicha asociación (Hermosillo), situación contraria al resto de los municipios donde se encuentran el resto de las OCV lo que permitió observar durante el trabajo de campo que la información pública desde sus portales no es visible dicha información.
Por último, no hay que perder de vista el Decreto del Presupuesto de Egresos que cada año se emite y que para el ejercicio fiscal 2019 el Ejecutivo del Estado redujo las asignaciones previstas 'transferencias a fideicomisos de entidades federativas y municipios en lo correspondiente a las Oficinas de Convenciones y Visitantes por \$23.3 millones de pesos' (Artículo 86 fracción I).

A continuación, se concentra en la siguiente tabla a los principales intervinientes en el proceso del flujo financiero del impuesto al servicio de hospedaje y su respectivo fideicomiso estatal. 
Tabla 2

Descripción de los responsables que intervienen en el proceso de flujo financiero del impuesto

\begin{tabular}{|c|c|}
\hline \multicolumn{2}{|r|}{ Sector Público } \\
\hline $\begin{array}{l}\text { Secretaria de } \\
\text { Hacienda Estatal } \\
\text { (SHE): }\end{array}$ & $\begin{array}{l}\text { Tesorería: Receptor-administrador del 2\% del Impuesto por la Prestación de Servicio } \\
\text { de Hospedaje (IPSH). } \\
\text { La Secretaría de Hacienda otorga el 90\% de los recursos del IPSH a las Oficinas de } \\
\text { Convenciones y Visitantes (OCV’s) y para la ciudad que no cuente con OCV se } \\
\text { transferirán los recursos a un fideicomiso creado por el ejecutivo estatal, y también a } \\
\text { la Comisión de Fomento al Turismo (COFETUR). Los recursos ejercidos de los } \\
\text { fideicomisos serán aplicados en promoción y publicidad turística. }\end{array}$ \\
\hline $\begin{array}{l}\text { SHE: Dirección de } \\
\text { Finanzas }\end{array}$ & $\begin{array}{l}\text { Pertenece a la Dirección General de Administración, quien se coordina con el analista } \\
\text { de información a cargo de vigilar el cumplimiento de contribuyentes sujetos al pago } \\
\text { del Impuesto por la Prestación de Servicio de Hospedaje. }\end{array}$ \\
\hline $\begin{array}{l}\text { La Comisión de } \\
\text { Fomento al Turismo } \\
\text { (COFETUR) }\end{array}$ & $\begin{array}{l}\text { Organismo público descentralizado con personalidad jurídica y patrimonio propios. } \\
\text { Tiene por objeto el fomento, promoción y desarrollo de las actividades turísticas en el } \\
\text { Estado. Ejerce o aplica el impuesto a través de un fideicomiso hacia la promoción } \\
\text { turística de aquellos municipios que no cuentan con OCV’S. A través de la Dirección } \\
\text { de Promoción y Eventos: Coordinar campañas de promoción y publicidad con } \\
\text { mayoristas hoteleros, agencias de viajes, oficinas de convenciones y visitantes del } \\
\text { Estado, entre otros, con el propósito de fomentar el turismo receptivo. }\end{array}$ \\
\hline Fideicomitente: & $\begin{array}{l}\text { Es la persona física o moral que aporta los recursos necesarios para operar el } \\
\text { fideicomiso (Gobierno del Estado). } \\
\text { Gasto público se integra por: fideicomisos públicos } \\
\text { Pagos: Los fideicomisos manejarán sus fondos y harán sus pagos a través de sus } \\
\text { propias unidades administrativas. } \\
\text { El fideicomiso presentará al Instituto Superior de Auditoría y Fiscalización (ISAF) } \\
\text { cada año, un informe financiero de la aplicación y destino de los recursos aportados } \\
\text { por el Gobierno del Estado durante el año inmediato anterior. } \\
\text { Rendición de cuentas } \\
\text { Se realizará un informe trimestral, el cual debe contener: } \\
\text { I.- Ingresos del periodo; } \\
\text { II.- Rendimientos financieros del periodo; } \\
\text { III.- Egresos del periodo y su destino; } \\
\text { IV.- Disponibilidades o saldo del periodo, y } \\
\text { V.- Listado de beneficiarios, en su caso. } \\
\text { La cuenta pública debe incluir un reporte del cumplimiento de la misión y fines de los } \\
\text { fideicomisos. }\end{array}$ \\
\hline
\end{tabular}




\section{Tabla 2}

\section{Continuación...}

\begin{tabular}{|c|c|}
\hline \multicolumn{2}{|r|}{ Sector Público } \\
\hline Fideicomisario & $\begin{array}{l}\text { Es la persona física o moral que recibe los beneficios de la operación del } \\
\text { fideicomiso (Gobierno del Estado). } \\
\text { Gasto público se integra por: fideicomisos públicos } \\
\text { Pagos: Los fideicomisos manejarán sus fondos y harán sus pagos a través de sus } \\
\text { propias unidades administrativas. }\end{array}$ \\
\hline $\begin{array}{l}\text { Comité Técnico del } \\
\text { Fideicomiso del Impuesto } \\
\text { al Servicio de Hospedaje }\end{array}$ & $\begin{array}{l}\text { El fideicomiso contará con un Comité Técnico } \\
\text { Integrado por: Un representante de Secretaría de Hacienda, uno de la Secretaría de } \\
\text { Economía, dos de la Comisión de Fomento al Turismo y siete vocales del sector } \\
\text { turístico del Estado (sector privado). }\end{array}$ \\
\hline Fiscalizadores & $\begin{array}{l}\text { ISAF: Instituto Superior de Auditoría y Fiscalización } \\
\text { Órgano de Fiscalización Superior del Congreso del Estado para realizar la } \\
\text { auditoría de los estados financieros, la revisión y fiscalización de la cuenta pública } \\
\text { del Estado y rendición de cuentas. } \\
\text { Fiscalización Superior: Facultad para conocer, revisar, auditar y evaluar el uso y } \\
\text { aplicación de los recursos públicos, de conformidad con las disposiciones } \\
\text { constitucionales y legales, a cargo del Instituto. }\end{array}$ \\
\hline \multicolumn{2}{|r|}{ Sector Privado Empresarial } \\
\hline Fiduciario & Es la institución bancaria autorizada para administrar los recursos del fideicomiso. \\
\hline Sector hotelero (SH): & $\begin{array}{l}\text { Forma parte del conjunto de unidades económicas agrupadas en el renglón de } \\
\text { alojamiento, clasificado por la industria turística. } \\
\text { Contribuyente u obligado fiscal en el traslado del impuesto a las personas a } \\
\text { quienes presta el servicio de hospedaje. Obligado fiscal en el entero del impuesto. }\end{array}$ \\
\hline $\begin{array}{l}\text { Prestadores de servicios } \\
\text { turísticos }(\mathrm{PT})\end{array}$ & $\begin{array}{l}\text { Las personas físicas o morales que ofrezcan, proporcionen, o contraten con el } \\
\text { turista, la prestación de los servicios dirigidos a atender las solicitudes a cambio de } \\
\text { una contraprestación. }\end{array}$ \\
\hline \multicolumn{2}{|r|}{ Organismo No Lucrativo } \\
\hline $\begin{array}{l}\text { Oficinas de } \\
\text { Convenciones y } \\
\text { Visitantes (OCV’s) }\end{array}$ & $\begin{array}{l}\text { Estructura que se encarga, en México, de la promoción de la oferta turística. } \\
\text { Figura jurídica que actúa como instrumento para ejercer el impuesto al hospedaje } \\
\text { en promoción turística (Asociación Civil); con el objetivo de posicionar } \\
\text { positivamente el destino turístico a través de un comité técnico que apoya e } \\
\text { impulsa el proceso de marketing, ventas, servicios y alianzas estratégicas para el } \\
\text { desarrollo y posicionamiento del destino }\end{array}$ \\
\hline
\end{tabular}


Tabla 2

Continuación...

\begin{tabular}{ll}
\hline Turista & \multicolumn{1}{c}{ Sociedad Civil } \\
\hline $\begin{array}{l}\text { Las personas que viajan temporalmente fuera de su lugar de residencia habitual y que } \\
\text { utilicen alguno de los servicios turísticos. En este caso el servicio de alojamiento, pagando } \\
\text { el impuesto del 2\% al hospedaje. }\end{array}$ \\
\hline $\begin{array}{l}\text { Impuesto al } \\
\text { servicio de } \\
\text { hospedaje }\end{array}$ & $\begin{array}{l}\text { La tasa del impuesto será del 2\% sobre el valor de las contraprestaciones que } \\
\text { los contribuyentes perciban por los servicios de hospedaje }\end{array}$ \\
\hline
\end{tabular}

Fuente: Elaboración propia con datos de la Ley de Hacienda del Estado, Ley General de Turismo, Ley del Presupuesto de Egresos y Gasto Público Estatal, Ley de Fiscalización Superior para el Estado de Sonora, Glosario de Coordinación de Planeación del Consejo de Promoción Turística de México y Reglamento interior de COFETUR.

\section{Conclusiones}

El haber analizado integralmente el contexto normativo del fidecomiso público, permitió identificar la correlación de obligaciones a cumplir por el mandato legal pero también por las obligaciones de informar por el modelo de transparencia que avanza para alinearse a mejores prácticas internacionales.

La principal conclusión de este estudio es que, para garantizar la transparencia del fideicomiso público estatal, resulta fundamental reconocer la aplicación de los marcos normativos vigentes interconectados como lo son en materia de Regulación Básica, Presupuestal y en materia de Transparencia y Acceso a la información.

Con este estudio se comprueba que son diversos los actores que directa e indirectamente se interrelacionan para la administración financiera de los recursos públicos, por lo que no debe verse al instrumento jurídico del fideicomiso de forma aislada, ya que el hilo conductor en este proceso es un recurso financiero catalogado como público o del gobierno, por lo tanto, la creación de la red permitió observar de manera más clara que se amplía el espectro de los sujetos obligados hasta abarcar a todo tipo de servidor público del ámbito federal, estatal y/o municipal, e incluso particulares (proveedores) u otros como la asociación Oficina de Convenciones y Visitantes, cuando manejen recursos públicos bajo cualquier modalidad.

Lo antepuesto, ya que durante el trabajo de campo se constató que las OCV tienen la idea de no informar el destino de los gastos, sobre todo en los municipios de la entidad federativa en estudio, lo cual fue posible identificar por las solicitudes de información al sector público que redirigían o declinaban lo solicitado o finalmente las evidencias eran enviadas por dichas OCV en pdf, ilegibles o denotando esfuerzo por atender lo solicitado.

Se comprueba el supuesto afirmativo de considerar el fideicomiso público como un nodo con necesidad de transparencia en la estructura de la red, ya que depende directamente de la responsabilidad de las partes intervinientes, de la calidad que la política pública es ejecutada y de si se logran resultados en la población objetivo; ya que actualmente se están incluyendo en la cuenta pública del estado reportes financieros relativos
Carrillo Cisneros, E., Ruiz León, A. A., e Ibarra Morales, L. E.
Vol. 7, núm. 19 / enero - abril del 2022

DOI: https://doi.org/10.36791/tcg.v7i19.116

Pp. 88-106 
al fideicomiso del impuesto al hospedaje y eso posible con la alineación de marcos jurídicos que interactúan (básica, presupuestal y de transparencia ) entre los diversos niveles de gobierno que se espera permitan homogeneidad y transparencia a las finanzas públicas estatales, ya que en años anteriores no se mostraba información al respecto en la cuenta pública estatal.

La consulta a diversos estudios en el entorno de la colaboración, partes involucradas y actores; con denotación de políticas públicas y abordados a través del análisis de redes, son de importante referencia como el de Maya y Alieva (2018), donde concluyen resultados derivados de cooperación entre actores clave y de relevancia público-privadas y asociaciones que promueven la colaboración entre países en el sector turismo y que puede verse facilitada por la participación de intermediarios, [..] para contribuir a la sostenibilidad de los mismos. Se esperan avances para próximos estudios relativos a la materia con la reforma a la Ley General de Transparencia y Acceso a la Información Pública con expectativas a maximizar el derecho de acceder a información, en el que se incluyen a partidos políticos, sindicatos, fideicomisos, instituciones de beneficencia e incluso individuos, debido a que se estableció un catálogo de obligados a informar el destino de los recursos públicos. Estudio de la Red de Gobierno Electrónico de América Latina y el Caribe expresan:

comienzan a filtrarse crecientes demandas de apertura, de transparencia en la gestión, de participación en la elaboración de políticas estatales, de rendición de cuentas y responsabilización por el uso de los recursos públicos, de evaluación y control ciudadanos de los resultados gubernamentales [..] abriendo paso una concepción bajo la denominación genérica de 'gobierno abierto’ (Oszlak, 2013).

Carrillo Cisneros, E., Ruiz León, A. A., e Ibarra Morales, L. E.
El aporte con este tipo de investigaciones genera información de procesos de políticas públicas estatales respecto a conocer en qué y cómo se ejerce el dinero público y observar si hay discrecionalidad en dichos procesos.

\section{Referencias}

Asociación Nacional de Hoteles y Moteles de México. (2014). Rendición de Cuentas respecto de las Contribuciones en el Sector Turístico en México. https://www.amhm.org/wpcontent/uploads/2018/11/8.1Informe_Rendicion_de_cuentas.pdf

Batagelj, V., y Mrvar, A. (2021). Pajek: Paquete de programas para el análisis y visualización de grandes redes. http://mrvar.fdv.uni-lj.si/pajek/

Carrillo, E., Ruiz, A., y Zizaldra, I. (2019). El Impuesto por la Prestación de Servicio de Hospedaje y su estudio a través del Análisis de Redes Sociales: Interdependencia en la promoción y publicidad turística. https://doi.org/10.5565/rev/redes.842

Hernández, R., y Mendoza, Ch. (2018). Metodología de la investigación: las Rutas cuantitativa, cualitativa y mixta. McGraw-Hill Interamericana Editores, S.A. de C. V. México.

Jung, T. (2010). Policy networks: theory and practice. En Osborne, Stephen (ed.): The New Public Governance? Emerging perspectives on the theory and practice of public governance (pp. 351-364). Oxon: Routledge. https://doi.org/10.4324/9780203861684

Kickert, W., Klijn, E., y Koppenjan, J. (1997). Gestión de redes en el sector público: hallazgos y reflexiones. En WJ KickertE. Klijn y JF Koppenjan (Eds.), Gestión de redes complejas: Estrategias para el sector público (pp. 167-191). SAGE Publications Ltd. https://www.doi.org/10.4135/9781446217658.n10 
Márquez, D. (2020). Los alcances de lo público: los derechos humanos como límite a la acción del gobierno y la extinción de los fideicomisos públicos. Hechos y Derechos, (57), mayo-junio 2020. Instituto de Investigaciones Jurídicas, UNAM.

https://revistas.juridicas.unam.mx/index.php/hechos-yderechos/article/view/14758/15788

Martínez, A. (2020). Diagnóstico sobre transparencia para el monitoreo de los Fideicomisos. Núcleo de Organizaciones de la Sociedad Civil. p. 170. Ciudad de México.

Maya, I., y Alieva, D. (2018). Caminos trazados: redes de itinerarios de los turistas rusos en Andalucía. Redes. Revista Hispana para el Análisis de Redes Sociales, 29(1), 76-91. https://doi.org/10.5565/rev/redes.745

Organización para la Cooperación y el Desarrollo Económicos. (OCDE, 2016). Bonturi Marcos. Datos Abiertos Gubernamentales en México. El camino hacia adelante. Directorado de Gobernanza Pública (GOV) de la OCDE. https://www.oecd.org/gov/digitalgovernment/OGD\%20Mexico\%20SPA\%20translation. pdf

Oszlak, O. (2013). Gobierno abierto: hacia un nuevo paradigma de gestión pública. Red de Gobierno Electrónico de América Latina y el Caribe - Red GEALC. Septiembre de 2013. https://www.oas.org/es/sap/dgpe/pub/coleccion5rg.pdf

Salvatierra, S. (2019). Fideicomisos en México. El arte de desaparecer dinero público. Fundar, Centro de Análisis e Investigación, A.C. https://fundar.org.mx/fideicomisos-en-mexico-el-artede-desaparecer-dinero-publico/
Suayeta, J. (2002). Las reformas a la ley general de títulos y operaciones de crédito. Nuevo e irresponsable atentado a la institución jurídica del fideicomiso. Revista de Derecho privado, nueva época, año I, (2), mayo-agosto de 2002, 123-164.

http://historico.juridicas.unam.mx/publica/rev/derpriv/c ont/2/leg/leg7.htm

\section{Leyes, Reglamentos, Acuerdos, Lineamientos}

Cámara de Diputados del H. Congreso de la Unión (2013). Consejo Nacional de Armonización Contable (CONAC). Acuerdo vigente que establece los Lineamientos que deberán observar los entes públicos para registrar en las cuentas de activo los fideicomisos sin estructura orgánica y contratos análogos, incluyendo mandatos. Diario Oficial de la Federación. https://dof.gob.mx/nota_detalle.php?codigo $=5283964$ \&fecha $=02 / 01 / 2013$

Cámara de Diputados del H. Congreso de la Unión (2021). Acuerdo por el que se armoniza la estructura de las cuentas públicas. Consejo Nacional de Armonización Contable (CONAC). https://www.conac.gob.mx/work/models/CONAC/nor matividad/NOR_01_11_002.pdf

Cámara de Diputados del H. Congreso de la Unión (2021). Constitución Política de los Estados Unidos Mexicanos (2021). http://www.diputados.gob.mx/LeyesBiblio/pdf/1_2805 21.pdf
Carrillo Cisneros, E., Ruiz León, A. A., e Ibarra Morales, L. E.
Vol. 7, núm. 19 / enero - abril del 2022 DOI: https://doi.org/10.36791/tcg.v7i19.116 Pp. 88-106 
Cámara de Diputados del H. Congreso de la Unión (2020). Decreto por el que se ordena la extinción o terminación de los fideicomisos públicos, mandatos públicos y análogos.

https://www.dof.gob.mx/nota_detalle.php?codigo=559 $1085 \&$ fecha $=02 / 04 / 2020$

Cámara de Diputados del H. Congreso de la Unión (2019). Decreto del Presupuesto de Egresos (2018). Boletín Oficial del Gobierno del Estado. Decreto número 9 del Presupuesto de Egresos del Gobierno del Estado de Sonora para el ejercicio fiscal 2019 Tomo CCII Número 51 Secc. III Lunes 24 Diciembre 2018 Capítulo IV De las Reasignaciones art. 86. http://www.boletinoficial.sonora.gob.mx/boletin/image s/boletinesPdf/2019/12/EE271220193.pdf

Cámara de Diputados del H. Congreso de la Unión (2021). Decreto del Presupuesto de Egresos del Gobierno del Estado para el ejercicio fiscal 2021. Apéndice L 'Fideicomisos'. http://www.congresoson.gob.mx:81/Content/Doc_leye s/Doc_569.pdf

Cámara de Diputados del H. Congreso de la Unión (2021). Ley de Disciplina Financiera de las Entidades Federativas y los Municipios (2021). http://www.diputados.gob.mx/LeyesBiblio/pdf/LDFEF M_300118.pdf

Cámara de Diputados del H. Congreso de la Unión (2021). Ley de Fiscalización Superior para el Estado de Sonora (2021). http://www.congresoson.gob.mx/Transparencia/Leyes\#

Cámara de Diputados del H. Congreso de la Unión (2021). Ley de Hacienda del Estado de Sonora. (20132021). http://www.congresoson.gob.mx:81/Content/Doc_leye s/Doc_429.pdf
Cámara de Diputados del H. Congreso de la Unión (2021). Ley de Instituciones de Crédito (2021). http://www.diputados.gob.mx/LeyesBiblio/pdf/43_200 521.pdf

Cámara de Diputados del H. Congreso de la Unión (2021). Ley de Transparencia y Acceso a la Información Pública del Estado de Sonora (2021). http://www.congresoson.gob.mx/Transparencia/Leyes\#

Cámara de Diputados del H. Congreso de la Unión (2021). Ley Federal de Transparencia y Acceso a la Información Pública (2021). http://www.diputados.gob.mx/LeyesBiblio/pdf/LFTAI P_270117.pdf

Cámara de Diputados del H. Congreso de la Unión (2021). Ley Federal de Presupuesto y Responsabilidad Hacendaria (2021). http://www.diputados.gob.mx/LeyesBiblio/pdf/LFPRH _200521.pdf

Cámara de Diputados del H. Congreso de la Unión (2021). Ley Federal de las entidades Paraestatales (2021). Ley Federal de las Entidades Paraestatales (2021).

http://www.diputados.gob.mx/LeyesBiblio/pdf/110_01 0319.pdf

Cámara de Diputados del H. Congreso de la Unión (2021). Ley General de Contabilidad Gubernamental (2021). http://www.diputados.gob.mx/LeyesBiblio/pdf/LGCG_ 300118.pdf

Cámara de Diputados del H. Congreso de la Unión (2021). Ley General de Títulos y Operaciones de Crédito (2021). http://www.diputados.gob.mx/LeyesBiblio/pdf/145_22 0618.pdf
Carrillo Cisneros, E., Ruiz León, A. A., e Ibarra Morales, L. E. 
Cámara de Diputados del H. Congreso de la Unión (2021). Ley General de Transparencia y Acceso a la Información Pública (2021).

http://www.diputados.gob.mx/LeyesBiblio/pdf/LGTAI P_130820.pdf

Cámara de Diputados del H. Congreso de la Unión (2021). Ley Orgánica del Poder Ejecutivo del Estado de Sonora (2021).

http://www.congresoson.gob.mx/Transparencia/Leyes

Cámara de Diputados del H. Congreso de la Unión (2021). Ley Orgánica de la Administración Pública Federal (2021).

http://www.diputados.gob.mx/LeyesBiblio/pdf/153_11 0121.pdf

Cámara de Diputados del H. Congreso de la Unión (2021). Lineamientos Generales para el Acceso a la Información Pública en el Estado de Sonora. http://transparencia.esonora.gob.mx/NR/rdonlyres/72B 00D41-8BDE-4BED-8B6A031F5FF837EC/252079/LineamientosGeneralesparael AccesoalaInformaci\%C3\%B3nP\%C3\%BAb.pdf

Cámara de Diputados del H. Congreso de la Unión (2021). Lineamientos Técnicos Generales para la publicación, homologación y estandarización de la información de las obligaciones establecidas en el Título Quinto y en la fracción IV del artículo 31 de la Ley General de Transparencia y Acceso a la Información Pública, que deben de difundir los sujetos obligados en los portales de Internet y en la Plataforma Nacional de Transparencia. https://www.transparencia.ipn.mx/Apoyo/SIPOT/LTG _DOF28122020.pdf
Cámara de Diputados del H. Congreso de la Unión (2021). Reglamento de la Ley de Presupuesto de Egresos, Contabilidad Gubernamental y Gasto Público Estatal (2021). http://transparencia.esonora.gob.mx/NR/rdonlyres/CB A2ED6D-1C16-4773-B976EB2032AF2A0A/78297/reglleydepptodeegrcontabgub erygp.pdf

Cámara de Diputados del H. Congreso de la Unión (2021). Reglamento de la Ley Federal de Presupuesto y Responsabilidad Hacendaria (2021). http://www.diputados.gob.mx/LeyesBiblio/regley/Reg _LFPRH_131120.pdf

Cámara de Diputados del H. Congreso de la Unión (2021). Relación de entidades paraestatales de la Administración Pública Federal (2020). http://www.dof.gob.mx/nota_detalle.php?codigo=5598 392\&fecha $=14 / 08 / 2020$

Secretaría de Hacienda y Crédito Público (2020). Cuarto informe trimestral sobre la situación económica, las finanzas públicas, la deuda pública y los activos del patrimonio estatal. Entidades Paraestatales. https://hacienda.sonora.gob.mx/media/200967/entidade s_paraestatales_4to_trim_2020_i.pdf

\section{Sitios web}

INFOMEX (Sistema de Solicitudes de Información Pública). (2020). http://infomex.sonora.gob.mx/

Mexicoevalua.org. (2021). No tapemos el pozo: el destino de los fideicomisos en proceso de extinción. https://www.mexicoevalua.org/no-tapemos-el-pozo-eldestino-de-los-fideicomisos-en-proceso-de-extincion/

Plataforma Nacional de Transparencia. (2020). https://consultapublicamx.inai.org.mx/vutweb/faces/view/consultaPublica.xhtml\#inicio
Carrillo Cisneros, E., Ruiz León, A. A., e Ibarra Morales, L. E. 